\title{
Effects of deep diving on the trachea of the leatherback turtle
}

\author{
Colm Murphy
}

Department of Civil \& Environmental Engineering, UCC

\section{Introduction}

This work is concerned with the effects of deep sea diving on the trachea (airway passage) of the leatherback turtle. Leatherback turtles are capable of diving to depths greater than 1,200 meters. Humans, in comparison, may only reach depths of around 30 meters unaided. It is believed that the response of the trachea along with its material properties plays a leading role in determining the depth that can be attained during a dive. The long term objective of this research is to investigate the response of the trachea of the leatherback turtle during deep dives (300-1250m). Questions remain as to the material properties from which the trachea is composed of and how exactly does the trachea respond as it undergoes a deep dive. Answering these questions will help not only to build a complete understanding of the leatherback's ability to dive to depths greater than $1,000 \mathrm{~m}$, but will also inform the study of deep diving in mammals, even including humans, whose competitive free diving has been extended beyond 200 metres in recent years.

\section{Definition of a Trachea}

The trachea or the windpipe is the tube-like passage through which organisms breathe air in and out. This process is commonly referred to as the respiratory system (Figure 1). The trachea begins in the neck at the larynx, which is more widely known as the voice box. It continues as a single airway until the point of bifurcation. This is the position at which it diverges into two separate tube-like structures called the bronchi. The bronchi, in turn, each subdivide into smaller passages which connect to the lungs. It is here that gaseous exchange takes place. The oxygen that is breathed in is transported through the trachea and the other airways into the lungs and converted to carbon dioxide. Carbon dioxide subsequently travels back up the trachea and is breathed out. In humans, the trachea consists of a number of c-shaped cartilage rings (Figure 2) that are connected to each other by a connective biological material. Cartilage is a firm and rubbery material that provides support and structure to biological matter, in this case the trachea. 


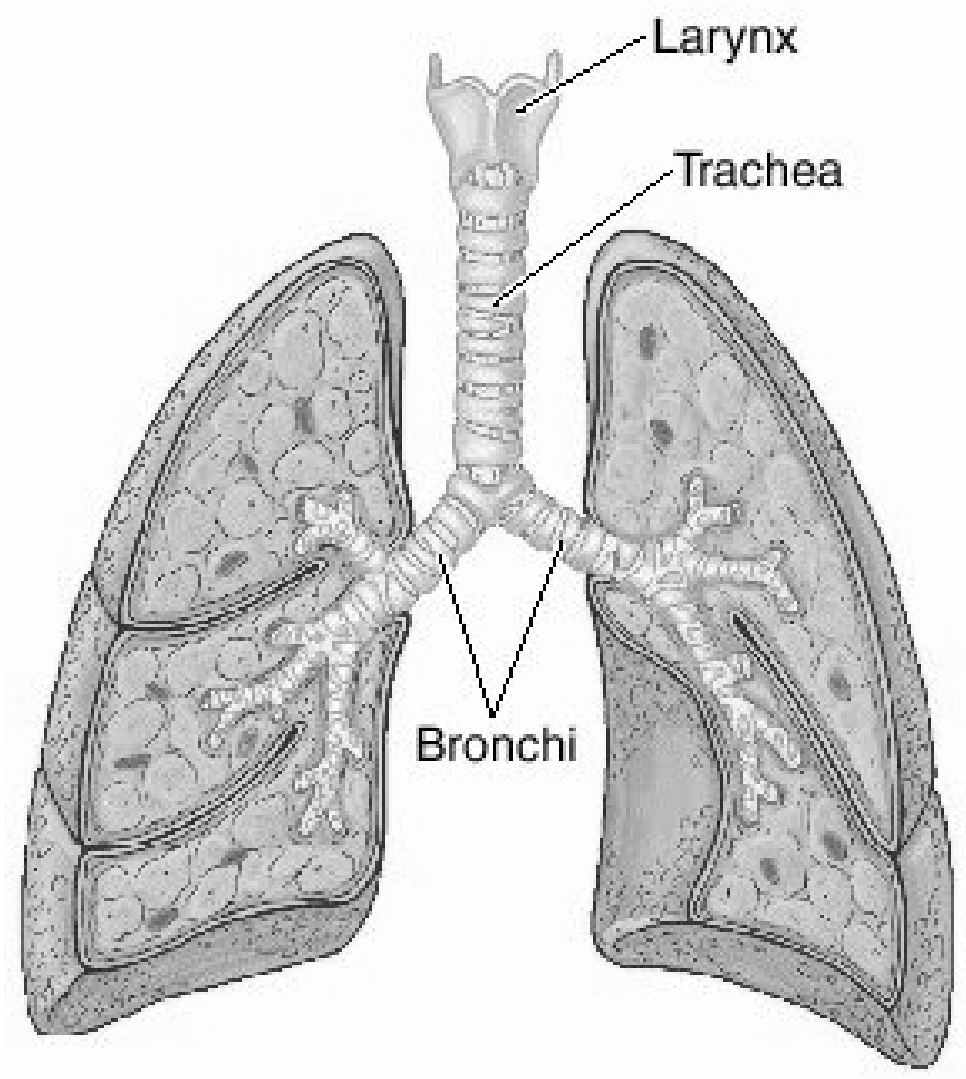

Figure 1: Respiratory System

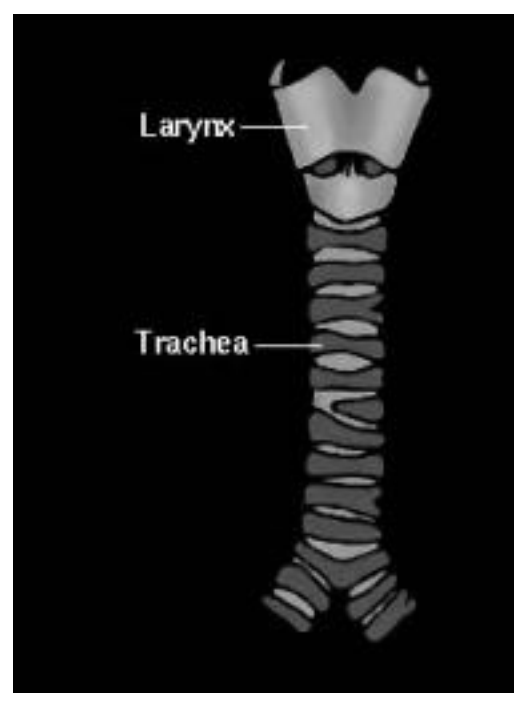

Figure 2: Composition of a typical human trachea 


\section{Deep Diving}

When any life form undertakes a dive, an external hydrostatic pressure is applied on that body. Hydrostatic pressure is the pressure applied by the surrounding water on a body and it increases linearly with depth. The deeper a body is in water the greater the hydrostatic pressure acting on it. A very important concern with diving is to avoid nitrogen decompression sickness, which is known more widely as 'the bends'. This occurs when a body is returning to the surface of the water. During this stage of the dive, it is exposed to decreasing hydrostatic pressures. These decreasing pressures cause the dissolved gases inside the lungs to change into bubbles which lead to much discomfort in the body and may even result in paralysis or death.

Humans are capable of diving unaided to around 30m before experiencing 'the bends'. The world record for deepest dive on scuba is $330 \mathrm{~m}$. In 2007 a navy diver set a record by submerging $610 \mathrm{~m}$ using an advanced hard shell suit. The leatherback turtle, however, is capable of diving up to $1,200 \mathrm{~m}$ naturally. How the turtle dives to these depths repeatedly and returns safely to surface level is not understood completely. It is hypothesised that the trachea collapses wholly or partially during dives. The trachea of the leatherback turtle differs in structure from that of Humans and also has different material parameters. Material parameters are the characteristic properties of a material which affect its strength and stiffness. Stiffness, in turn, is a measure of the resistance of a material to an applied force. The trachea of the leatherback turtle is composed of near complete elliptical rings (as opposes to c-shaped rings in Humans) of hyaline cartilage with only small amounts of intervening connective tissue. The resulting pipe-like structure has sufficient stiffness to remain open for respiration and has a high rotational stiffness that allows for limited neck movements.

\section{Methodology}

The research to date this has been concerned with classifying the material parameters of the tracheal cartilage of the leatherback turtle. In order to do this a sample of a leatherback trachea was compressed in the laboratory (Figure 3(a)). The testing conformed to all biomechanical standards and was set up to replicate the response of the trachea as it is subjected to increasing hydrostatic pressures in a typical dive sequence. A model of the trachea was created using a structural analysis software program with the same testing details as that in the laboratory (Figure 3(b)). A basic rubber representation was deemed sufficient for modelling the trachea. The results from both sets of data (namely the force applied and the corresponding movement of the trachea at that time) were recorded. To run an analysis of a model the parameters of the material in the model must be declared. How the model responds to applied forces depends on its material properties. As the 

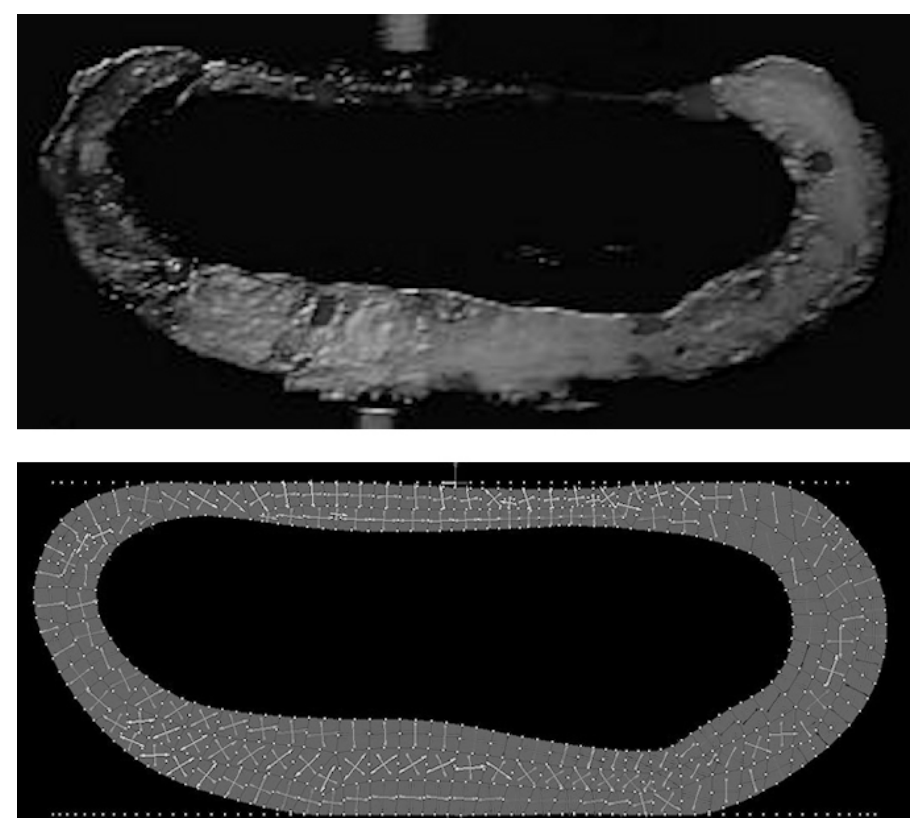

Figure 3: (a): [Upper] Trachea deformed in the laboratory test at time $t=140$; (b): [Lower] Trachea deformed in the model at corresponding time

aim of this part of the research is to find out these parameters, it is hypothesized that by continuously running this model with differing values for the parameters, at some stage the response of the trachea in the model will match that of the response in the laboratory. This process is known as optimisation. Basically this involves providing initial 'guess' values as well as upper and lower boundary values for the parameters. The optimisation process changes the values accordingly until a prescribed error or tolerance is satisfied. In this case, the desired outcome is that the error between the model results and the laboratory results is minimised. This concept of finding out the material properties (or input parameters) from output responses is also referred to as an inverse method.

\section{Results}

Figure 4 shows the plots of the experimental and final model results for the optimisation process. In both cases it is a graph of applied force versus displacement (or movement).

\section{Discussion}

This research demonstrates the viability of using an inverse methodology to classify the non-linear material properties of tracheal cartilage. The graphs of forces versus displacement in Figure 4 show good agreement between the experimental and model data. How closely these curves match is an indication of the accuracy of the methodology. The gaps 


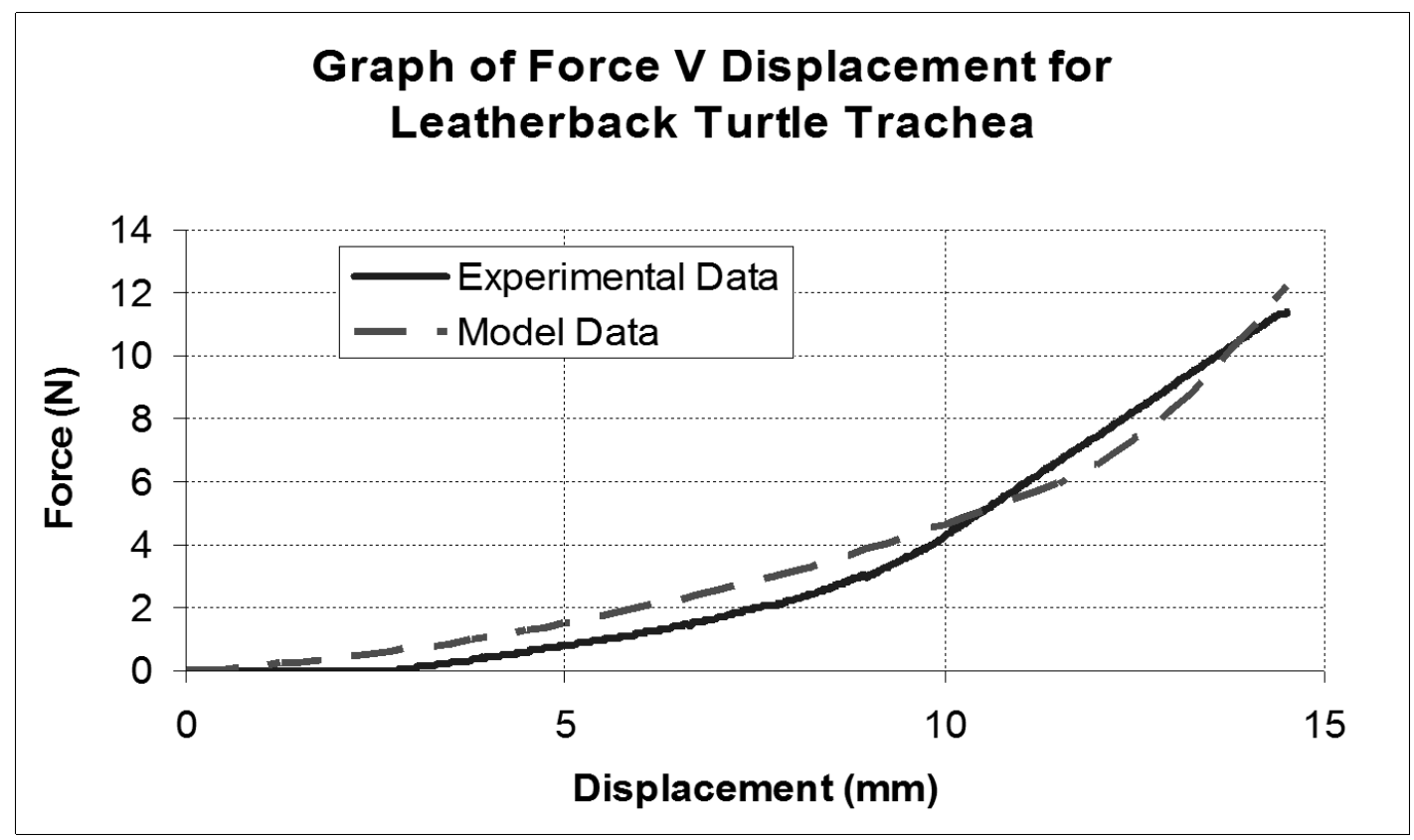

Figure 4: Plots of force versus displacement for the leatherback turtle trachea for the experimental and model data.

represent the error between the laboratory testing and the model. Means to resolve this error are currently being investigated. It is thought that by improving the geometry of the model and by considering more complicated material models better results shall be obtained. Work is also progressing on a two dimensional model of the neck section (Figure 5 (a) and (b)). This model shows how the entire neck section of the trachea responds due to increasing pressure in dives. The trachea is surrounded by the other materials in the neck of the leatherback turtle. In this model muscle is modelled as an incompressible rubber which is deemed sufficient for purpose.

Early findings from this research confirm the hypothesis that the leatherback trachea collapses (Figure 5 (b)) as it subjected to increased hydrostatic pressure in dives. Humans are unable to collapse the trachea to this extent due to different material properties of the trachea. Future work shall focus on improving the accuracy of the methodology discussed earlier and improving the models. Once the material parameters of the leatherback trachea have been established, a three dimensional model of the entire neck section shall be developed. This model shall provide a detailed description of how the trachea responds at any time and depth during a typical dive sequence. The results will, firstly, assist zoologists researching the diving behaviour and capability of the leatherback turtle and, secondly, provide insights into the application of structural engineering analyses and methodologies to finding solutions to biomechanical problems.

Colm Murphy is a student in the Department of Civil \& Environmental Engineering under the supervision of Dr. Denis Kelliher. This research is being carried out in collaboration with Prof. 

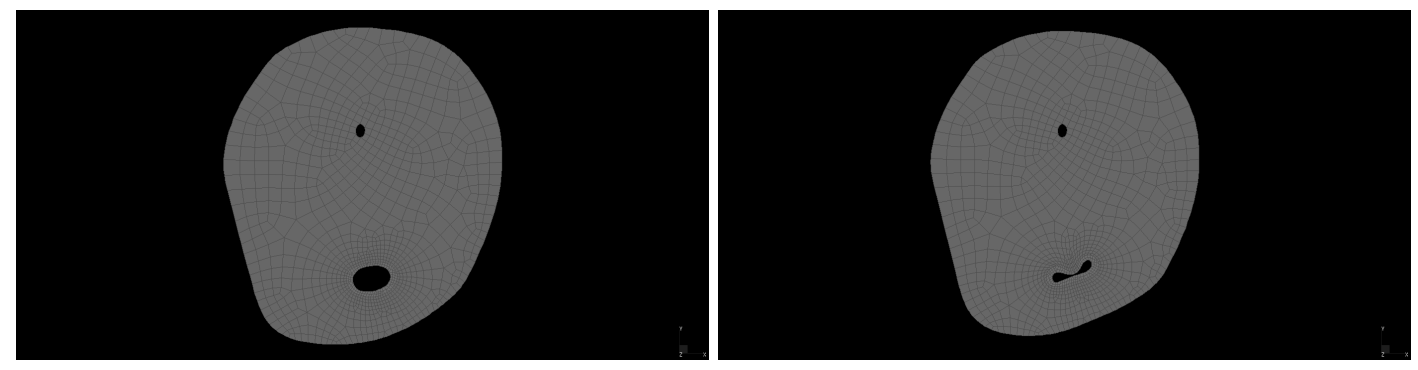

Figure 5: (a) [left] 2-D neck section in original position; (b) [right] 2-D neck section at $29 \mathrm{~m}$ depth

John Davenport in the Department of Zoology, Ecology and Plant Sciences. The author would like to acknowledge funding from the Irish Research Council for Science, Engineering and Technology (IRCSET) through their EMBARK initiative. 